\title{
Teaching by research at undergraduate schools: an experience
}

Manuel Costa

Manuel F. M. Costa, "Teaching by research at undergraduate schools: an experience," Proc. SPIE 3190, Fifth International Topical Meeting on Education and Training in Optics, (8 December 1997); doi: $10.1117 / 12.294387$

Event: Fifth International Topical Meeting on Education and Training in Optics, 1997, Delft, Netherlands 


\title{
Teaching by research at undergraduate schools. An experience.
}

\author{
Manuel F. M. Costa
}

\author{
Universidade do Minho, Departamento de Física \\ Tel. +35153 604327(/20); Fax. +35153678981 \\ Email: mfcosta@fisica.uminho.pt \\ P-4709 BRAGA CODEX PORTUGAL
}

\begin{abstract}
On this communication I will report a pedagogical experience undertaken in the 1995' class of Image Processing of the course of Applied Physics of the University of Minho. The learning process requires always an active critical participation of the student in an experience essentially personal that should and must be rewarding and fulfilling. To us scientists virtually nothing gives us more pleasure and fulfillment then the research processus. Furthermore it is our main way to improve our, and I stress our, knowledge.

Thus I decided to center my undergraduate students' learning process of the basics of digital image processing on a simple applied research program. The proposed project was to develop a process of inspection to be introduced in a generic production line. Measured should be the transversal distance between an object and the extremity of a conveyor belt where it is transported. The measurement method was proposed to be optical triangulation combined with shadow analysis. To the students was given almost entire liberty and responsibility. I limited my self to assess the development of the project orienting them and point out different or pertinent points of view only when strictly necessary.
\end{abstract}

Keywords: Teaching by research, University, Triangulation, Fresnel diffraction, Industrial automation and process control.

\section{A FEW REMARKS ON THE MAJOR PROBLEMS ON TEACHING SCIENCE AT UNDERGRADUATE SCHOOLS.}

Most of the problems affecting undergraduate applied optics' teaching at the university are common to many other fields specially those of science and technology ${ }^{(1-4)}$.

There are different points of view for these issues. Apart from the point of view of the community, that in the end should define and assess the teaching quality requirements and requirements' tolerances, three contributions are of major importance to this short analysis: the university; the teachers; and the students. The diagnostic and proposed solutions made by the different parts do coincide in significant extent. 
The university needs to give a positive answer to society demands. Not always these are clearly stated or stable. Specially with the industry the interaction is not always easy. Keeping a high quality teaching standard becomes extremely demanding in financial terms. It is permanently necessary the scientific and pedagogical actualization of educators and support staff, the replacement and improvement of laboratories and equipment for pedagogical support in order to cope with the rapidly changing requirements. Another important problem facing the university is the numerus clausus and the obligation of preparing in quantity and due time students upon the market needs and political determinations.

From the point of view of the teacher apart of the evidence of a social and professional depreciated position, the main problems come from the work conditions: in terms of work load; pedagogical and scientific actualization conditions; deficiencies in the laboratories and support pedagogical equipment and staff; but also the student's behavior in general terms ${ }^{(1)}$. At this level the low high school preparation specially in mathematics and Portuguese is a basic major constraint. Major difficulties of communication (either in terms of oral, written or plastic expression or comprehension) are noticed at alarming levels. Important is also to notice that this is recognized by the majority of student as the major constraint to the development of their studies. We have made in 1995 an informal inquest ${ }^{(1)}$ on students' opinions about the quality of the course, and their own performance and expectations. Statements produced here about students' point of view take into account the inquest's results.

The lack of motivation is another major issue justified by the students (eventually not deeply enough) by social distress and absence of perspectives for the future (employment, security...).

The need of hard work is definitely difficult to accept by the students(we are the fast food generation ...). They tend to act very passively. No sense of enjoying learning is the norm. Over $80 \%$ of our students think that the clear explanation, be the teacher, of the subjects is by far more important than their own participation on the analysis of questions and problems. Moreover the students think the teacher should not expect or demand an active participation of the students on class.

Nevertheless, it is exactly on the opposite direction we (teachers) should focus our efforts: encouraging or even demanding an active permanent participation of the students; trying to develop their sense of observation (seeing critically) and critical reasoning by performing more experimental work and bringing to discussion real life issues (an extreme caution must to be taken here to stress clearly the limitations of our often simplified approaches); demanding hard work and stimulating the sharing of ideas (communication). Trying to make the students to be predisposed to have fun in class it's rather important. Although not always easy to implements this is an effort that may give a good result's improvement.

Finally one should never forget to adapt our teaching behavior to the particular conditions (social, student status or situation, community requirements,...) that condition our work, but never compromising in terms of our basic principles as teachers and teachers of science.

\section{TEACHING BY RESEARCH}

On the lines presented above was developed the work presented in this paper.

\subsection{Introduction}

At the second semester of the academic year of 1995/96 I had in my class of Image Processing ${ }^{(1,5)}$ a small homogeneous group of four students. Furthermore they also have been my students at the Fiber Optics classes in the previous semester. So, they were already familiarized to have in class an active, critical spirit, responsible, demanding, self-learning and self-rewarding posture. The basic conditions were gathered to try my pedagogical experience: "Teaching by research" (in fact the title should rather be "Learning by research" as we will see 
below). Thus I divided the 14 weeks of the course in three parts. In the first six weeks several lectures were made introducing all the basic subjects involved in the theme of image characterization, acquisition and digitalization, and digital image processing techniques. Practical examples were frequently introduced.

Four practical works were done at the laboratory taking around 12 hours. Different software was used to make the students work and learn all the major image processing operators applied on the images the students them self chose and digitized. At the end of this period the students knew the essential of all available hardware and software equipment available. Then three practical problems were presented to the students: early detection of skin cancer by the detection of skin signs its characterization (size, shape, roughness, color distribution...), storage and automated comparison with previous acquired images of the same marks; distance measures using video cameras and image processing; cars' speed measures by image acquisition, digitalization and treatment.

During two weeks they discuss the possible solutions to each one of them. The process was freeze for a while and the main problem/project was defined. The problem was specified as follows: in one conveyor belt running at a fixed low velocity, are dropped, at a known low frequency, small cubic boxes. The boxes fall always with faces aligned with the belt, but at different, erratic, transversal distances from the sides of the conveyor belt. The method to be developed should deal with the determination of that lateral position.

During over five weeks the student used each one of the six hours per week (and surely some more...) trying to understand and solve the problem.

At the end one full extended week was enough to settle all the fundamental image processing issues in a systematic way.

We should never forget teaching takes time. Learning takes much more. Increasing students' specific knowledge is important. But above all science demands work, responsibility and method. It is precisely this the science teaching should give to the students. Perhaps more important than knowing concepts or theories is to know how to get them. It's necessary to think, to reason, to analyze critically and interactively problems and situations. More important then the final concepts, is knowing, feeling, to interiorize the paths or processes of, or to, understand them.

\subsection{Specification of Students' Task}

The practical assignment is specified more closely as presented below. The students were asked to develop a method for assessing the lateral position of 0.1 meters large' cubic boxes dropped at a known frequency into one 0.45 meters wide conveyor belt running at a fixed low speed. The boxes fall always with faces aligned with the belt, but at different, erratic, transversal distances from the sides of the conveyor belt.

Optical triangulation ${ }^{(7-14)}$ was suggested as the basis for the method that also should integrate 2D image' data processing and feature's detection with subpixel resolution. The theoretical study of dual camera passive triangulation was considered. This was perhaps the best approach on view of the pedagogical objectives of the work. Nevertheless a one camera configuration was chosen also on view of the available equipment, by economical considerations and system's robustness.

In order to study the best possible resolution the system can give, no movement should be involved. No conveyor belt movement is present. The box is displaced laterally, perpendicularly to the conveyor belt's movement direction. The lateral position is closely controlled by the employment of a precision linear displacement stage. However it is always important to have in mind that a good industrial system is one that fits the requirements reliably within the given tolerances ${ }^{(15)}$. Thus the students have to realize the importance of the 
influence of the movement and all other practical implementation constraints on the system's resolution, accuracy and reliability.

\subsection{Optical Triangulation}

By its simplicity, robustness and reliability, optical triangulation is the major method employed to measure distance or depth in situations like ours. Depths of several hundred millimeters have to be measured with resolutions in the millimeter or submillimeter range.

Depth measurements are done for long time using different optical triangulation based procedures ${ }^{(7-14)}$ : moiré methods that combines interferometry and triangulation; contrived lightning where a structured pattern or simply one light stripe (that is made to scan the sample by mechanical or opto-mechanical means) is projected onto the object at a known angle and is viewed by one or two cameras from another(s) angle(s); and discreet passive or active triangulation employing one or more cameras or detectors with eventually more than one light source. Comparative studies on triangulation based dimensional inspection methods are presented elsewhere ${ }^{(7-9)}$.

Figure 1.

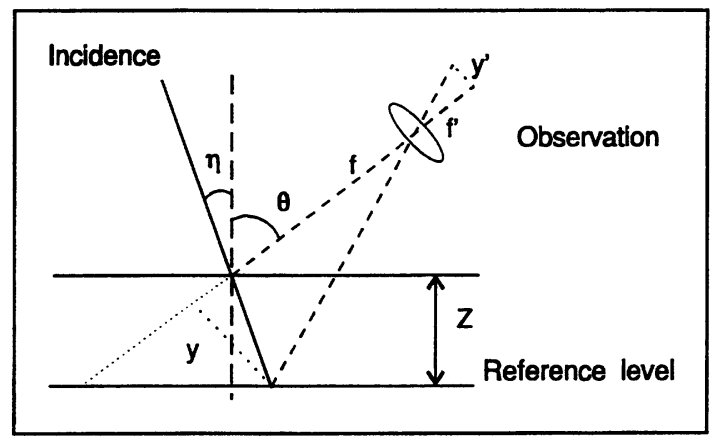

Figure 1. The general triangulation geometry.

On Figure 1. the general triangulation procedure is sketched. The relation between the measured value $\left(y^{\prime}\right)$ and height $(Z)$ comes:

$$
y^{\prime}=M \frac{Z \sin (\eta+\theta)}{\cos \eta+(Z / f) \cot (\eta+\theta)}
$$

where $M=f / f^{\prime}$ is the optical magnification in the observation arm, $f$ and $f^{\prime}$ are the focal lengths of the observation optics, $\eta$ the incidence angle and $\theta$ the observation one.

\section{A BRIEF DESCRIPTION OF OUR METHOD.}

Figure 2. illustrate in a simple way our sensing procedure. A point like light source is placed side to side with a CCD camera at a known distance. Both are aligned with one side of the conveyer belt. Opposite is placed a white smooth target. Being point like the light source can be figured as an isotropic source of light rays that hit the target at different angles. In the top of Figure 2. is presented a plot of the profile of the irradiance distribution (in arbitrary scale) in the target plane. No background lightning exists and there is no reflections in any objects in the surroundings. No diffraction effects are considered just the geometrical optics $\left(1 / r^{2}\right.$ variation, being $r$ the distance from a target point to the point source) approach. When the box pass in front the light source a shadow is projected onto the target. The shadow's edge location on the target's plane depends on the box position $(h)$. 
The target is imaged into the CCD' sensor. The acquired image is digitized and the edge's location obtained. A look-up table (LUT) obtained by calibration is then used to calculate $h$.

Figure 2.

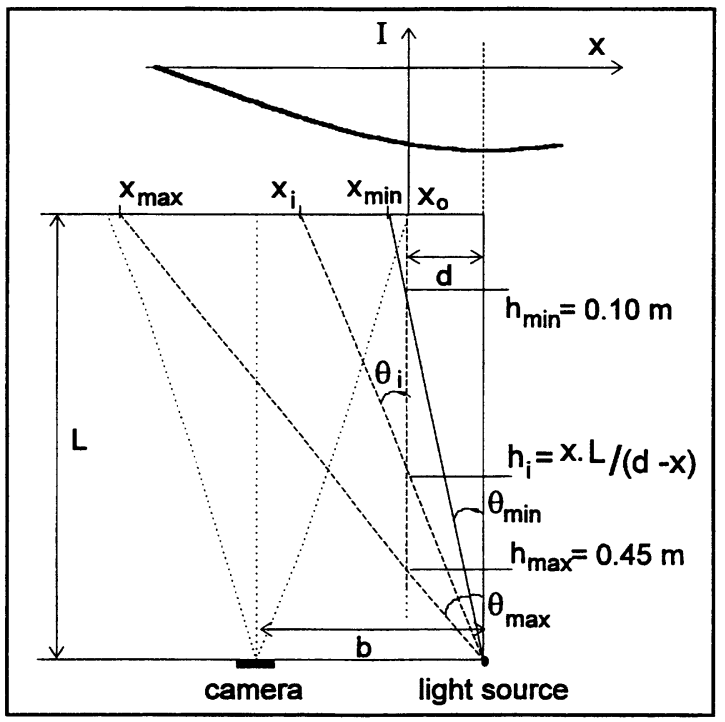

Figure 2. Our measurement procedure.

\subsection{The shadow analysis.}

The problem of shadow formation has been deeply studied for long time. Is for all known that in a shadow there is never a perfectly sharp transition from the bright to the dark side. It depends on the way the obstacle is illuminated, the obstacle's type and also the type of target where the shadow is projected onto.

Figure 3.

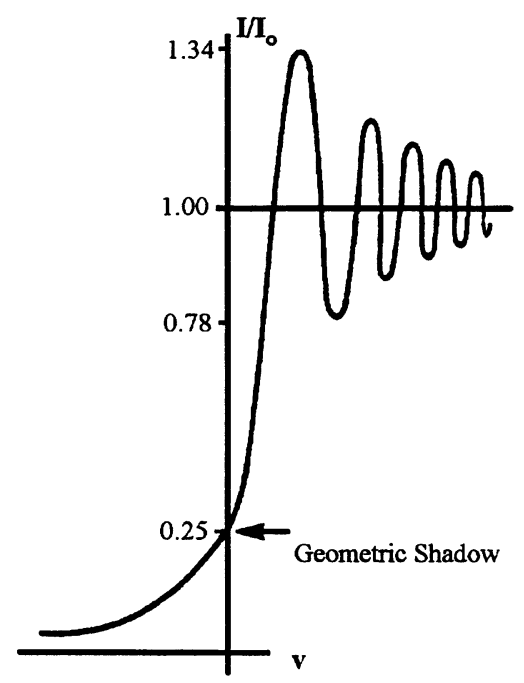

Fig. 3. Fresnel diffraction. Straight edge's shadow. 


\subsubsection{Fresnel diffraction.}

The shadow's edge location is the one geometrical optics will indicate. The major reason is the diffraction effect on the obstacle's edge. In our case we are in the regime of Fresnel diffraction. Details can be easily found elsewhere. Let us just to show (Figure 3.) the profile of the rate of the shadow's intensity distribution to the undisturbed (without obstacle) intensity for this kind of diffraction regime. It can be seen that there is some light within a geometric shadow although falling monotonically to zero. Also at the geometrical shadow the intensity is already reduced to $25 \%$ to the undisturbed intensity. Outside the shadow the intensity rises to more than its undisturbed value and oscillates with increasing frequency and reducing the amplitude until it reaches the undisturbed value.

Figure 4 .

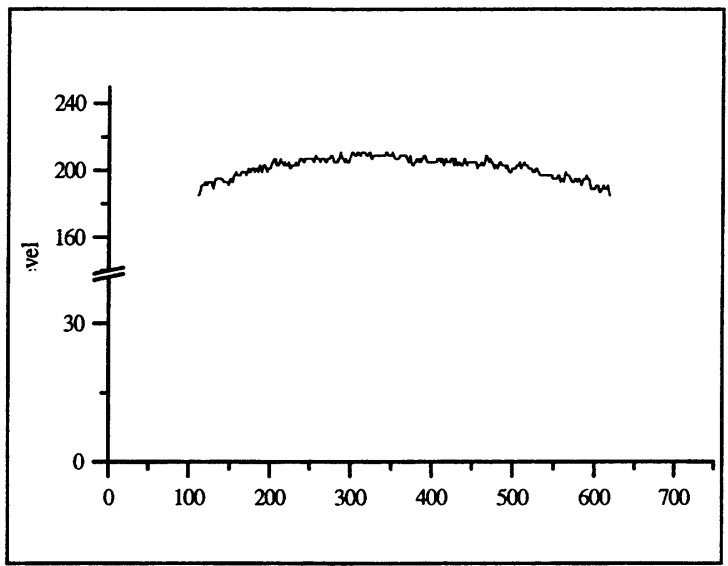

Fig. 4. The rms of the noise (after subtraction of the baseline) of the undisturbed target's irradiance profile is 2.6 pixels.

Figure 5.

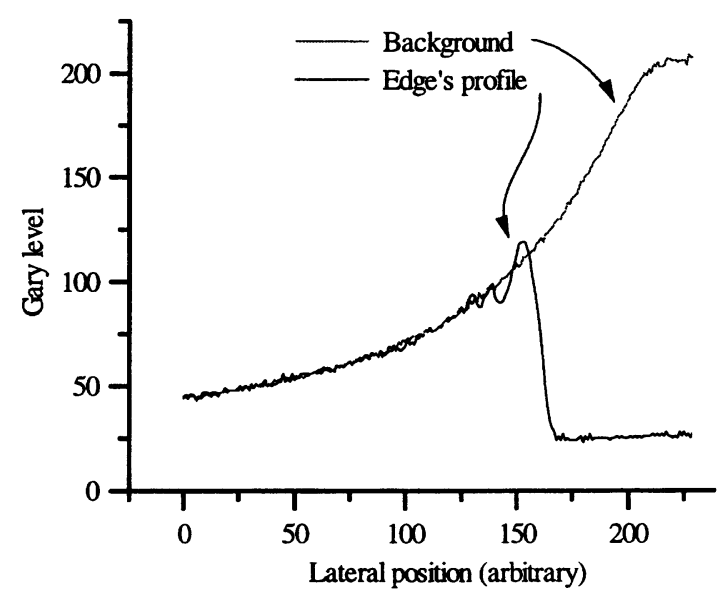

Fig. 5. Shadow's against background's profile. 
In Figure 5. and 6. such an edge is presented. We would like to stress that usually the obtained profiles do not show that clear oscillating structure. In fact the high frequency noise from the detection's opto-electrical conversion and electronics is added to image.

The rough texture of the target used is also responsible for those rather noisy profiles (Figure 4. and 5.). Furthermore the point spread function of the camera's objective and sensor will convolve with target's intensity profile resulting in a sort of dilution (distortion) of image's details ${ }^{(5,6)}$. (In figures 7. and 8. the Fourier transforms of shadow's and undisturbed profiles are shown). Nevertheless the obtained results seem to fit well the theory.

Figure 6.

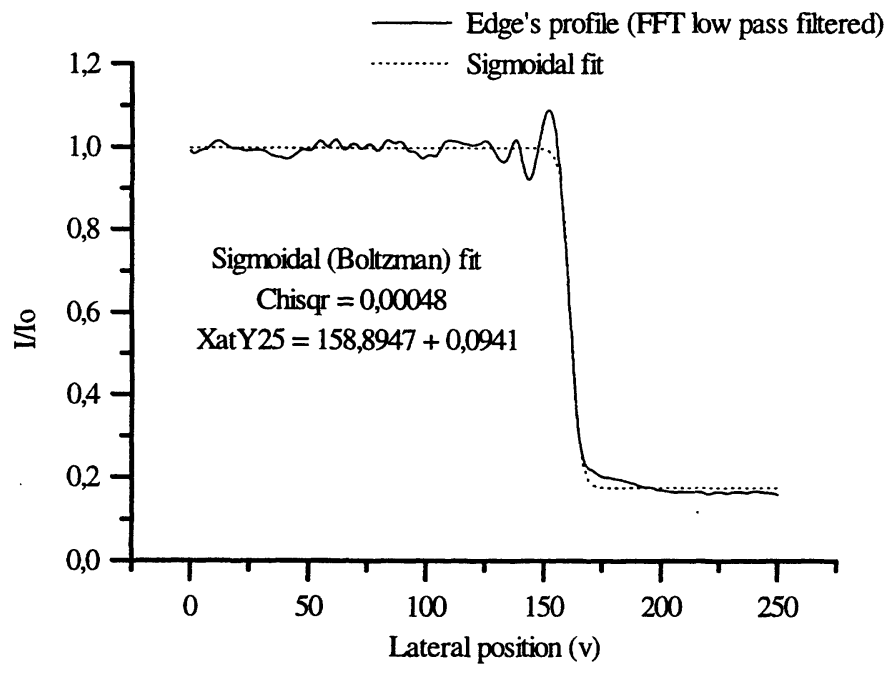

Fig. 6. Fitting a Boltzman function to the shadow's edge.

Figure 7. and 8.
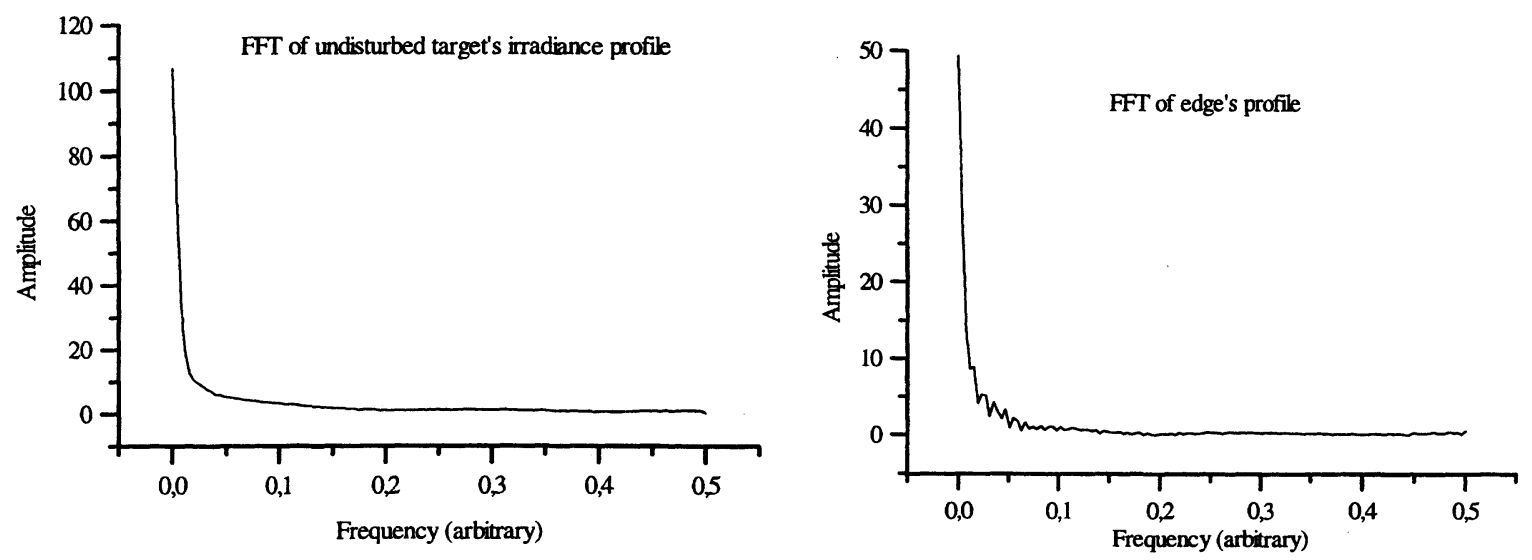

Fig. 7 and 8. The frequency content of the background's profile and of shadow's profile (right). 
Figure 9.

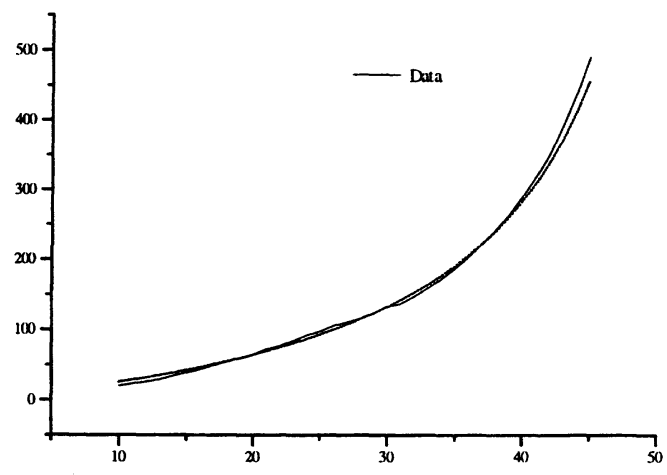

Fig.9. Calibration curve obtained with no subpixel interpolation.

Figure 10.

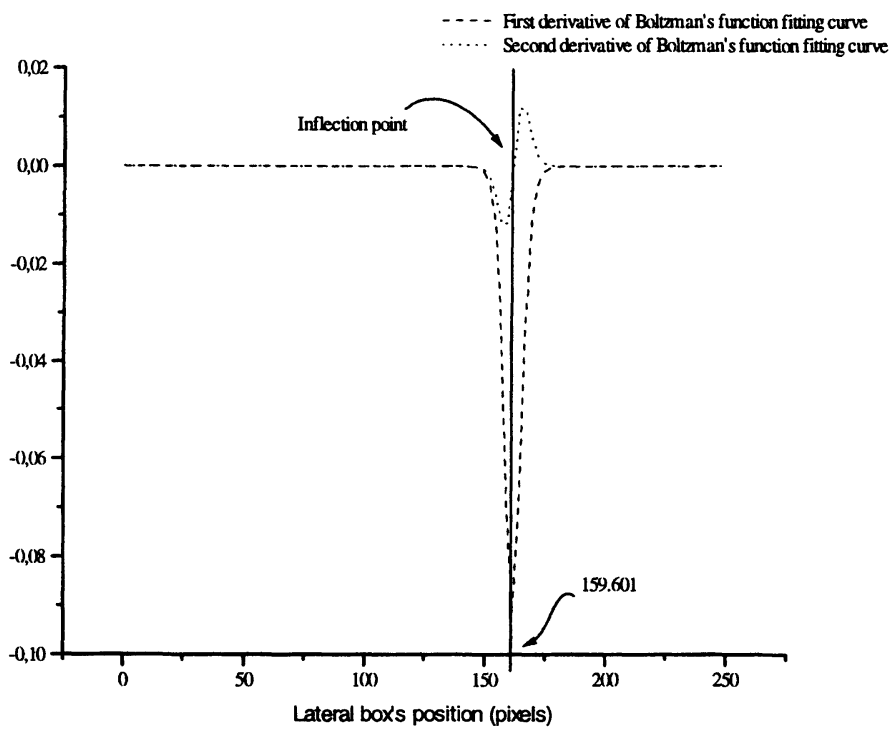

Fig. 10. The edge located by the inflection point.

\subsubsection{Shadows' edge location}

On the above we decided to perform the shadow's edge location in the following steps: the undisturbed profile is subtracted from the shadow's image profile (Figure 6.); a sigmoidal fitting on that is performed; the geometrical shadow position is then determined at subpixel resolution $\left(I / \mathrm{I}_{0}=0.25\right)$.

In order to proceed with the shadow's edge location the digitized images may be processed in different ways: histogram equalization normalizing the maximum and minimum gray level values for all images (the illumination on the target varies with shadows' location); low pass Fourier filtering to eliminate high frequency 
noise; band pass Fourier filtering in order to isolate the "Fresnel oscillations"; morphologic operators in order to reduce the region of bright to dark shadow's transition; edge detection operators however for the degree of resolution we want the shadow's edge location to have may hardly give good results in these situations.

From the digitized image the shadow's edge location can be performed in many different ways. The easier one is to define a gray level threshold and to find the pixel that presents a gray level closer to the threshold. In Figure 9. we present the results of the systems' calibration obtained by defining a threshold that is $25 \%$ of the mean gray level of the undisturbed target's irradiance profile (Figure 4.). Eventually the edge may be located at subpixel resolution by interpolation between the adjacent two (four,...) pixels with gray levels closer (in each side) to the threshold. However to perform the interpolation one should know the form of the distribution of gray levels along the shadow's edge area (Figure 5. or 6.). A Boltzman function seems to fit very well the profile. Hachicha et al. ${ }^{(16)}$ suggested to fit a suitable function to the gray level edges' profile and to find its inflection point (Figure 6. and 10.).

\section{SYSTEM'S CONFIGURATION.}

Several system's configurations were considered. In Figure 11. is presented the relation between the shadow's edge location on the camera's sensor plane and the distance from the point source to the target. Experimental data is plotted (dots) over the theoretical curve for a box depth of $h=20 \mathrm{~cm}$. The relation between the shadow's edge location and $d$ is linear. Unfortunately the relation between the measured quantity $x$ and box's position or depth, $h$, is not linear. If we decided to use a light source placed far apart from the conveyor belt the ray illuminating the scene will be parallel and the conversion relation $(x$ to $h)$ will be simple and linear (ignoring aberrations, misalignments and non uniformity at the sensor). Nevertheless keeping all hardware closer will certainly be more convenient. Thus the choice of a point like source was clear. On view of the problem' constraints and camera's characteristics we decided to make $L=56.5 \mathrm{~cm}, \mathrm{~d}=9.00 \mathrm{~cm}$ and $\mathrm{b}=40 \mathrm{~cm}$. Thus $\mathrm{x}_{\min }=1.974 \mathrm{~cm}$ and $\mathrm{x}_{\max }=35.217 \mathrm{~cm}$. The displacement of the box's shadow edge between the extreme positions in the conveyor belt will roughly correspond on the detector plane to almost a 500 pixel's shift of the image's shadow edge location. This means that virtually the whole available sensing camera's area will be used thus allowing the best resolution.

Figure 11.

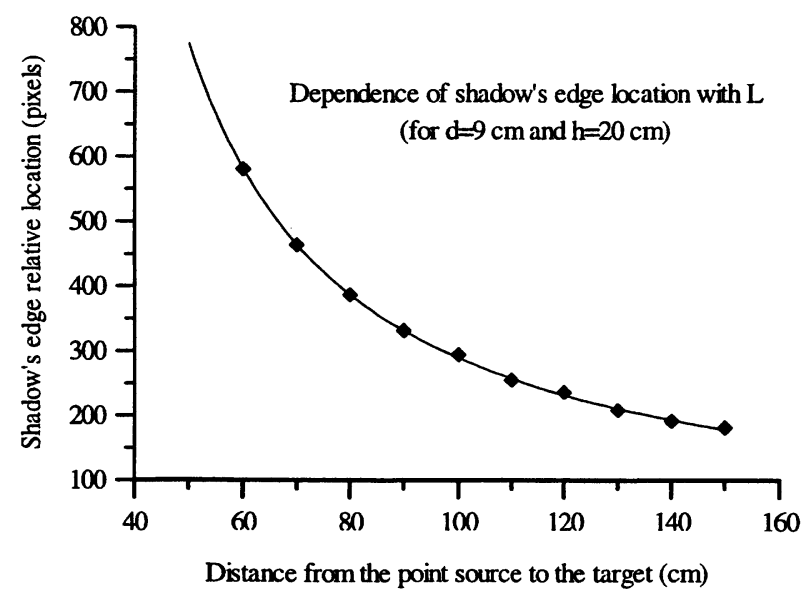

Fig. 11. Shadow's edge location versus $\mathrm{L}$. 
The light source used is a simple $20 \mathrm{~W}$ bulb. The CCD camera is an Electrim's EDC-1000HR b/w camera supplied with a "frame-grabber" computer interface card. The sensor with $753 \times 244$ (488 interlaced) pixels have a sensing area of $8.67 \times 6.59 \mathrm{~mm}^{2}$. The exposure time can be controlled from $10 \mathrm{sec}$. down to $1 \mathrm{msec}$. The objective used is a Computar of $8.5 \mathrm{~mm}, 1: 1.5$ and 2/3" diameter. The system presents some aberrations including barrel distortion. Thus we set the system in such a way that the pixels on the extreme areas of the sensor are not used. Nevertheless the calibration procedure can deal with these problems in large extent.

\subsection{Calibration}

The calibration procedure is rather simple. A sample box is aligned in the system and is made to give consecutive precise displacements (the positioning error was of $0.1 \mathrm{~mm}$ for the calibration reflected on Figure 3 . and of $0.01 \mathrm{~mm}$ for the one of Figure 12.). For each position, $h$, the shadow's image is digitized and the edge located as explained above. As stated before the calibration curve obtained with the employment of the sigmoidal fitting in order to achieve subpixel resolution approaches quite well the theoretical curve. Ten calibration curves were obtained by this method. The average curve is plotted in Figure 12. compared with the theoretical curve. As one could expect, although the measured data fits overall well the theoretical ones, the measurements' accuracy and resolution decrease significantly for higher depths (roughly above $25 \mathrm{~cm}$ ). In Figure 13. the errors on the edge location at different box's depths are plotted.

Figure 12. and 13.

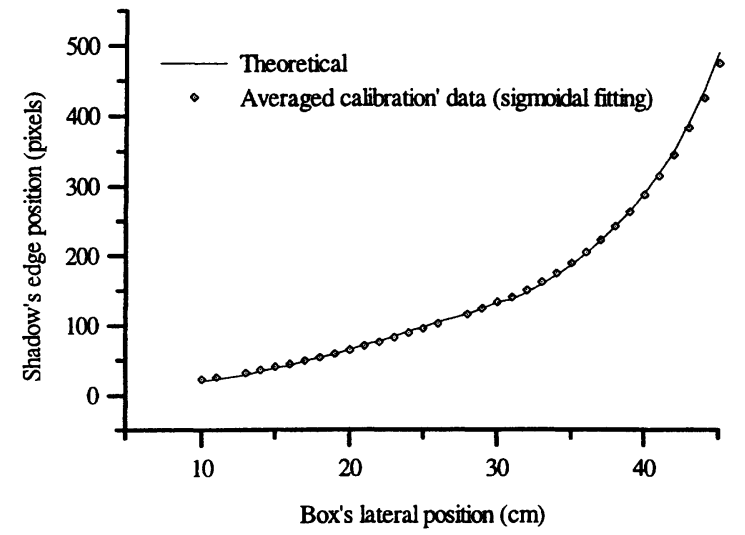

Fig. 12. The accepted system's calibration curve.

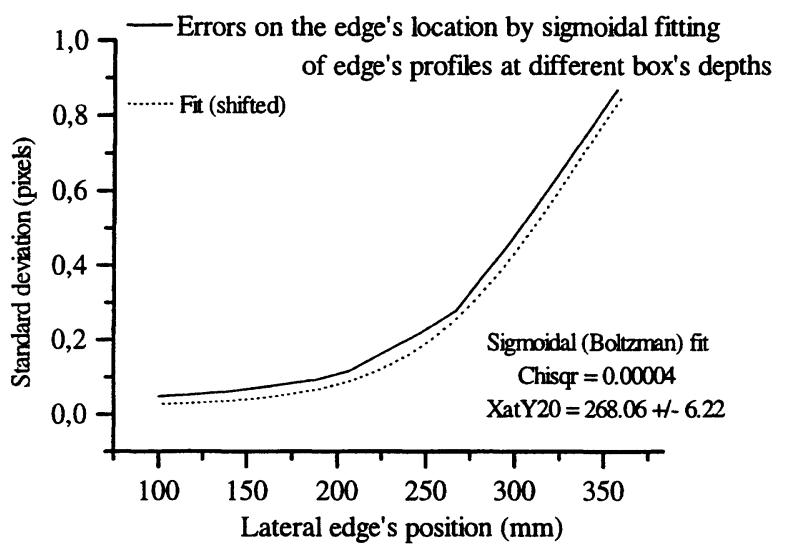

Fig. 13. The calibration errors.

\section{CONCLUSION}

The process of teaching image processing should be essentially practical. The contact with the analysis and solution of specific practical, simulated or real life, problems is in this context fundamental. Furthermore image processing is a strongly interdisciplinary activity. Apart from a good knowledge of the fundamental techniques of image digitalization and processing is necessary a deep understanding of the, in this case physical, problems and processes involved in situations where image processing procedures have to be introduced. The students also have to be aware of the specific constraints and requirements presented by the industrial world. The problems should be solved fast within the requirements and requirement's tolerances always looking for solutions low cost, reliable, robust and friendly in use preferentially in an automated way. The evolution in quality and price on image acquisition, digitalization and processing procedures is leading to a wide spread use of those in industrial' quality control. 
The simple solution to our very simplified specific problem despite its limitations could well find some practical use in a production line. Depth measures up to several ten centimeters with resolution of down to some microns is possible by applying subpixel interpolation techniques upon the knowledge of the physical origin of the optical shadows. Further work is needed to extend the application of this method to more general real world situations (different conveyor belts' speed and size box's sorting, non constant dropping frequencies and erratic box's misalignments).

However, from the pedagogical point of view the project gave excellent results. In what concerns the learning of image processing and image acquisition and digitalization, but also on the students' character building. They do realize the importance of reliability and responsibility, and improved their work method gaining self-confidence and self-esteem.

\section{ACKNOWLEDGMENT}

For their work in this project the author wish to express his acknowledgment to Mr. Jorge, Faria, Carvalho and specially Mr. Las Casas, students of the 1995's class of Image Processing of the course

(licenciatura) of Applied Physics (specialization in Optics and Lasers) of the Physics Department of the University Of Minho.

\section{REFERENCES}

1. Manuel F.M. Costa, "Teaching Applied Optics at The University of Minho", Proc. Soc. Photo-Opt. Instrum. Eng. 2525, 357-361 (1995).

2. P. Narayana Swany; "Put the real world in the physics curriculum", letter, Physics Today, vol. $48, \mathrm{n}^{\circ} .9$, p. 15 (1995).

3. A. S. Prasanis; "Teaching Optics in the Optics Age", Proc. Soc. Photo-Opt. Instrum. Eng. 2525, 318-330 (1995).

4. Shoang C Donn; "Optics Education for Now and Future from an Entropy Perspective", Proc. Soc. Photo-Opt. Instrum. Eng. 2525, 497-507 (1995).

5. Introdução ao processamento de imagem, Manuel F.M. Costa, Universidade do Minho, 1995.

6. Digital Image Processing, W. K. Pratt, John Wiley \& Sons, 1978.

7. Microtopografia laser, Manuel F.M. Costa, Universidade do Minho, 1993.

8. Manuel F.M. Costa, J.B. Almeida; "System of optical noncontact microtopography", Applied Optics, vol. 32, $n^{\circ} .25,4860-4863$ (1993).

9. Manuel F.M. Costa, "Surface Inspection by an Optical Triangulation Method", Opt. Eng. 35(9) 2743-2747 (1996)

10. Manuel F. M. Costa, J. B. Almeida; "Surface relief mapping", in Proceedings of the International Conference on Industrial Inspection, D. W. Braggins, ed., Proc. Soc. Photo-Opt. Instrum. Eng. 1010, 193-199 (1988).

11. H. Takasaki, "Moiré topography", Appl. Opt. 9, 6, 1467 (1970).

12. Jae S. Lim, M. S. Chung; "Moiré topography with color gratings, Appl. Opt. 27, 13, 2649-2650 (1988).

13. M. Halioua, F. P. Chiang; "Projection moiré with moving gratings for automated 3D topography", Appl. Opt. 22, 850 (1983).

14. Y. Takagi, S. Hata; "High speed precise 3-D vision sensor using slit-light method", Proc. IEEE Int. workshop on industrial applications of machine vision and machine intelligence, 235-239 (1987).

15. R. A. Brook, "Automatic inspection in industry today", in Proceedings of the International Conference on Industrial Inspection, D. W. Braggins, ed., Proc. Soc. Photo-Opt. Instrum. Eng. 1010, 2-7 (1988).

16. A. Hachicha, S. Simon; "Subpixel edge detection for precise measurements by vision systems", in Proceedings of the International Conference on Industrial Inspection, D. W. Braggins, ed., Proc. Soc. Photo-Opt. Instrum. Eng. 1010, 148-157 (1988). 